\title{
Inmigrantes en formación: Estado de bienestar y promoción de lazos sociales en el caso de la formación para la inserción laboral de las personas inmigrantes en Barcelona \\ Immigrants training: welfare state and promotion of social bonds in the case of workforce training programs for immigrants in Barcelona
}

\author{
Laura Cristina Yufra \\ Universidad Autónoma de Barcelona, laurayufra@yahoo.com.ar
}

\section{Resumen}

\section{Palabras clave}

Estado

Inmigración

Formación para la

inserción laboral

\begin{abstract}
La tesis constituye un recorrido teórico-empírico en torno a la configuración sociopolítica del fenómeno de la inmigración como "problema de Estado" a partir del estudio del caso de la formación para la inserción laboral. Se señala la intrínseca relación entre inmigración y Estado y se identifican los procesos de transformación en la promoción de los lazos sociales por parte del llamado Estado "de bienestar". En el desarrollo del trabajo se analiza el acceso a los recursos de formación de las personas inmigrantes donde resulta cuestionada la idea de la obtención progresiva de derechos sociales para inmigrantes. Los contenidos de los cursos de formación apuntan a la conformación del inmigrando deseado: se espera que las personas inmigrantes se adapten, y sean sujetos disponibles y activos, que dejen de lado todas las "particularidades" (culturales, sociales, etc.) que son entendidas como rémoras para la inclusión exitosa en la sociedad de instalación.
\end{abstract}

\section{Abstract}

\section{Keywords}

State

Immigration

Workforce training
This dissertation presents a theoretical-empirical analysis of the socio-political construction of the immigration phenomenon as a "state issue" starting from the study of the case of workforce training programs. First of all, it points out the intrinsic relationship between immigration and State, and how the latter is still a decisive factor in the treatment of the former. Besides, it identifies the transformation processes in the promotion of social bonds by European welfare states, which affect the provision of resources and social rights aimed at immigrants. In this respect, the commonly accepted idea of a progressive access to social rights by immigrants is questioned. The contents of the training courses are also aimed at producing of a wanted immigrant: immigrants are expected to adapt themselves, to be available and active subjects and to put aside all their "peculiarities" (cultural, social, etc.), which are seen as a hindrance for a successful inclusion in the installation society.

\section{De puntos de partida}

Uno de los principales puntos de partida de esta tesis doctoral se encuentra en la casi omnipresente y estereotipada comprensión de la inmigración como un "problema social", lo que hace necesaria una revisión de la reducción que supone atribuir los desajustes y conflictos que acaecen en una determinada sociedad a la presencia de personas inmigrantes. Es así que lo primero que se emprendió fue la construcción de un marco teórico crítico que apuntara a considerar que las personas inmigrantes no plantean estrictamente "nuevas" problemáticas a las sociedades en las que se establecen sino que más bien ponen de manifiesto los límites y tensiones que ya se encontraban presentes en ellas. En este

\footnotetext{
Yufra, Laura Cristina (2013). Inmigrantes en formación: Estado de bienestar y promoción de lazos sociales en el caso de la formación para la inserción laboral de las personas inmigrantes en Barcelona. Athenea Digital, 13(2), 301-309. Disponible en

http://psicologiasocial.uab.es/athenea/index.php/atheneaDigital/article/view/Yufra
} 
sentido, se revela totalmente ingenua la pretensión de ciertos actores sociales de dar por sentado que con anterioridad a la llegada de los sujetos inmigrantes las sociedades no estaban amenazadas en su cohesión social, su solidez económica o su homogeneidad cultural.

Así pues, en este marco teórico se entrelazaron los aportes de autores como Pierre Bourdieu (1999a; 1999b; 2001; 2012), Abdelmalek Sayad (2010) y Andrew Geddes (2006), Andrew Geddes y Michael Bommes (2000) con el abordaje de la Antropología de las políticas de Chris Shore y Susan Wright (1997) para analizar los lineamientos y las prácticas de intervención en el campo de la formación para la inserción laboral de las personas inmigradas. El aparato crítico construido apuntó a señalar el carácter inextricablemente relacional que se da entre la inmigración y la sociedad de instalación. Por lo tanto, aquella no puede ser considerada como un fenómeno exógeno a la sociedad de establecimiento de las personas inmigradas ni como prescindible; sino que, lejos de esto, cada sociedad de instalación, a partir del tratamiento que hace de la presencia de las personas inmigradas, configura "su" propia inmigración. Además, y precisamente debido a este carácter ineludiblemente relacional, la inmigración se constituye como un analizador fundamental de los procesos que afectan y atraviesan a las sociedades en las que las personas inmigrantes se instalan.

\section{Principales anclajes}

En el actual orden geopolítico neoliberal y globalizado, las personas que emigran son categorizadas como inmigrantes por parte del Estado-nación en el que se establecen, un Estado-nación que continúa poseyendo un rol sociopolítico fundamental, que entre otras cosas hace "nacer" o "alumbra" a la inmigración como tal. En este sentido, la definición de quién es "extranjero", así como el control de los flujos migratorios, sigue siendo una potestad ejercida por los Estados-nación. Ahora bien, debido a esta condición de extranjería estatalmente constituida se solicita imperativamente a las personas inmigrantes que se "integren" socialmente. Sin embargo, no se puede obviar que las políticas de control migratorio generan poderosas categorías sociales que condicionan sobremanera las posibilidades que las personas inmigrantes tienen a unas buenas condiciones de vida, al trabajo y a los recursos asistenciales. Así como tampoco se puede obviar que, en ocasiones, las denominadas políticas de integración vienen simplemente a subsanar situaciones de fragilidad y de vulnerabilidad que han sido provocadas por las propias políticas de control de los desplazamientos migratorios.

El hecho de que la tesis se haya centrado específicamente en el ámbito de las políticas de integración y de formación para la inserción laboral, ha servido para enfocar la reflexión en las maneras en las que actualmente se están promoviendo los lazos sociales que configuran la pertenencia a una determinada sociedad. En concreto, el ámbito de la formación para la inserción laboral es identificado como un ámbito privilegiado a través del cual es posible la movilidad y el ascenso social. La gran importancia otorgada a este tipo de formación se fundamenta en el supuesto de que una persona, a través de seguir un proceso formativo, podrá acceder a un mejor puesto de trabajo y así mejorar su propia situación socioeconómica.

Para contextualizar tales políticas de formación para la inserción laboral de las personas inmigrantes fue ineludible poner en relación y discusión dos ámbitos temáticos que suelen ser habitualmente abordados por separado. Por un lado, aquellas teorías e investigaciones que están abordando de manera crítica las transformaciones que el Estado de Bienestar está padeciendo, es decir aquellos trabajos que se ocupan del desacoplamiento de la ligazón entre pertenencia social y el goce igualitario de derechos sociales, y, por otro, aquellos estudios y reflexiones que se centran en los procesos de integración de las personas inmigrantes. 
A este respecto, existen algunos antecedentes en lo que podemos considerar un campo emergente de estudios sobre la relación entre inmigración y Estado de bienestar en España, que es donde hemos circunscrito nuestra investigación. Las maneras de abordar esta relación tienen mucho que ver con las preocupaciones propias y dominantes de la sociedad de instalación que la llevan a preguntarse en qué medida los recursos del Estado de bienestar constituyen un atractor, o lo que se ha popularizado como un "efecto llamada", de las migraciones. En este sentido, Rosa Aparicio y Andrés Tornos (2002) han indagado sobre la relación entre Estado de bienestar e inmigración poniendo el acento en el papel que juegan las representaciones que los inmigrantes se hacen del Estado de Bienestar español. Asimismo algunos autores han investigado sobre las diferentes maneras en que los inmigrantes realizan aportes al Estado de bienestar (Moreno Fuentes y Bruquetas Callejo, 2011). Esta gran preocupación por calcular los costes y beneficios de la inmigración, más allá de la conclusión habitual de que es multidimensionalmente beneficiosa, difunde la idea de que el valor de algo depende de la utilidad o de las ganancias, que de ello se pueda obtener. Por otro lado, hay que insistir en que también existen algunas contribuciones en torno a las políticas sociales en ámbitos concretos, como puedan ser la educación, la sanidad, la vivienda y su relación con la inmigración (Cachón, 2009; Cachón y Laparra, 2009; Clavijo y Aguirre, 2002; Garde, 2000).

En contraste con estos enfoques, la perspectiva crítica que hemos elaborado en nuestra investigación nos ha permitido interrogarnos sobre cómo el actual Estado "de bienestar", mediante los diversos recursos que ofrece (o niega), configura la inmigración como deseada o indeseable.

A partir de esta interrogación fue posible reconocer y explicitar los criterios de deseabilidad en cuanto a las formas de pertenencia a la sociedad en la que se instalan las personas inmigrantes. De este modo, fue también posible pensar los procesos culturales, políticos y socioeconómicos de inclusión/exclusión, ya sea de los propios ciudadanos o de los ajenos, que acontecen en las sociedades contemporáneas a partir del análisis de los procesos de intervención formativa y sociolaboral con las personas inmigrantes.

Desde una perspectiva metodológicamente cualitativa se optó por la realización de entrevistas abiertas y semidirectivas a coordinadores de formación, a formadores y a usuarios de aquellas entidades laboralmente formativas en las que se realizó el trabajo de campo, así como por la consecución de observaciones participantes en dichas entidades, teniendo en cuenta la máxima variedad organizativa. Así se fue construyendo un mapa de recursos formativos heterogéneo y fluctuante, a partir de preguntas que giraban principalmente en torno a cómo era el acceso a la formación y a cuáles eran los contenidos que dicha formación dirigida a las personas inmigrantes tenía.

\section{Hallazgos teóricos más destacables}

Uno de los aportes principales de la tesis consistiría en haber subrayado el carácter sociopolítico del fenómeno de la inmigración, frente a las comprensiones economicistas y culturalistas predominantes, y en haber destacado el papel de los Estados en la configuración de la inmigración a través del "pensamiento de Estado" y de la trama institucional en el que se concreta dicho pensamiento. En este sentido, el cruce de las fronteras territoriales sigue siendo un indicador extraordinariamente relevante, pues pone de manifiesto cómo los poderes del Estado-nación no se encuentran, como con demasiada frecuencia se suele sostener, en su ocaso.

\footnotetext{
${ }^{1}$ Entrecomillamos la expresión "de bienestar" para referirnos a la forma actual en la que se organiza la distribución de provisiones sociales, que no cumple las características que hicieron posible el desarrollo socio-histórico del llamado Estado de bienestar entre mediados de los años '40 y mediados de los años '70 del siglo pasado.
} 
Además habría que apuntar el hecho de haber reconocido cómo los poderes de los Estados-nación se ejercen a través tanto de la definición del estatus jurídico de sus miembros, como mediante la configuración de los Estados de "bienestar", que establecen fronteras organizativas al ofrecer (o al negar) el acceso y disfrute de derechos y recursos sociales. Los Estados "de bienestar" actuales funcionan como imperceptibles fronteras internas que diseminan sutilmente la idea (de enormes y graves consecuencias políticas) de que existen usuarios "legítimos" e "ilegítimos" de los recursos de los que el Estado dispone. Tales fronteras organizativas establecen y refuerzan las actuales dinámicas exclusógenas que conducen fundamentalmente a las personas inmigrantes (pero no sólo a ellas) a situaciones de extrema vulnerabilidad.

Por lo que hace al análisis de las políticas sociales, cabe destacar el abordaje conjunto de las "políticas de integración" y de las "políticas de control" migratorios, evitando así dar una versión unilateral de aquellas primeras, ya que ambas se influyen mutuamente. La consideración en exclusiva de las políticas de integración resta elementos explicativos ya que, muchas veces, estas políticas vienen a paliar situaciones generadas por las políticas de control de los flujos migratorios.

\section{Resultados teórico-empíricos más remarcables}

Entre los hallazgos teórico-empíricos más reseñables cabría destacar, en primer lugar, el que la provisión de cursos de formación depende estrechamente del estatus legal de las personas inmigradas. De esta manera, las personas sin permiso de residencia y/o de trabajo son quienes poseen un acceso más limitado a todo tipo de servicios sociales y un suministro "informal" de prestaciones que son ofrecidas por organizaciones no lucrativas de la sociedad civil y al margen de las otorgadas por las administraciones públicas. Por consiguiente, existe una gran inestabilidad y desobligación en la promoción de la formación por parte del Estado, al dejar en manos de otros actores la asistencia a las personas inmigrantes en situación administrativa irregular; una asistencia que está generalmente ligada a la caridad y que es siempre precaria.

Por su parte, existen recursos de formación destinados específicamente para personas que tienen un permiso de residencia vinculado al de un familiar. Tales cursos refuerzan los espacios donde existe una selección previa del mercado como "trabajos para inmigrantes" y están caracterizados también por una escasa valoración social. Además, al otorgarse dichos recursos sólo a quienes se encuentran recién llegados, se parcializa y fragmenta la intervención social dirigida a las personas inmigrantes. De esta forma, se define de modo cada vez más estricto quienes son los beneficiarios legítimos de determinados derechos sociales (así como quienes no tienen ningún derecho a ello).

Ahora bien, los recursos normalizados de los que hacen uso las personas inmigrantes, también tienen un sesgo diferenciador en la medida en que el permiso de trabajo está estrechamente ligado a las necesidades del mercado laboral. En este sentido, resulta cuestionada la idea comúnmente aceptada de la obtención progresiva de derechos sociales: el panorama se presenta sinuoso y desparejo, mediado por el acceso a recursos sociales y por las limitaciones propias del Estado "de bienestar", y en particular del Estado de bienestar español. Por lo dicho, no se puede hablar de un acceso "universal" a los cursos de formación o de que se garantice el ingreso en igualdad de condiciones a las personas inmigrantes.

En segundo lugar, los resultados teórico-metodológicos ponen de relieve la importancia que las características organizativas de la formación tienen para la inserción laboral. Así, el desarrollo reciente y muy limitado del Estado "de bienestar" español se puede identificar en un ámbito como el de la formación para la inserción laboral que presenta un gran nivel de complejidad por su heterogeneidad, multiplicidad 
de actores y finalidades entrecruzadas. En dicha complejidad, emergen las tensiones organizativas que este modelo contiene.

La formación, que se presenta básicamente sustentada en subvenciones, es percibida como "gestión" (es decir, como un terreno técnico y administrativo) que no deja lugar para el reconocimiento de posibles conflictos entre los distintos actores que intervienen en dicho proceso, ni para el desarrollo de las especificidades de éstos. Por este motivo, las entidades formativas presentan la dificultad de establecer prioridades en las líneas de actuación y los contenidos formativos.

Otro resultado remarcable consiste en la hipervisibilización y magnificación de la ayuda destinada a las personas inmigrantes, lo que se refleja ilustrativamente en la recurrida expresión de "les damos todo". Sin embargo, y como contrapartida, se produce un ocultamiento o invisibilización de las ayudas que se otorgan a las empresas a través, por ejemplo, del servicio de selección del personal formado o, incluso, de la contratación de formadores vinculados a empresas específicas para que realicen la formación laboral a la medida de éstas.

No insistiremos nunca lo suficiente en que estos dos inseparables procesos tienen una importante incidencia en la percepción social de la distribución de los recursos.

Asimismo el trabajo hizo posible reconocer cómo la formación puede estar más próxima a los intereses de las empresas que deciden (sobre todo en la formación continua) a qué formación pueden asistir sus empleados y quiénes son las personas que se formarán. Pero además, no es raro que las necesidades de las empresas se limiten a competencias muy básicas de sus empleados. Esto es incompatible con el derecho de las personas que buscan formarse a obtener una mejora en sus condiciones de vida a través de la obtención de trabajos mejor remunerados y más valorados socialmente.

Esto hace pensar que la importancia que se suele atribuir a la formación como vía para la superación de las desigualdades tiene un carácter retórico antes que constituir un objetivo alcanzable con una clara voluntad política.

Por otra parte, en la actividad cotidiana de las entidades formativas se constituye la figura del inmigrado, de tal manera que se espera que éste sea un sujeto totalmente disponible y activo, donde la formación (y el trabajo que se conseguirá tras la misma) es un recurso que hay que "merecerse". Las personas, por consiguiente, tienen que despojarse de las rémoras de todo tipo: culturales, lingüísticas o familiares, para poder adaptarse adecuadamente al mercado laboral.

En consecuencia, las políticas y las prácticas de formación de las personas migradas, en tanto que política activa para el empleo, pueden inscribirse en el marco de la paulatina difusión de una reformulación de los criterios de pertenencia social y de la posibilidad de gozar de tales recursos sociales en base a determinados merecimientos antes que en función de los derechos de ciudadanía.

La formación para la inserción laboral se perfila como un dispositivo que antes que servir para la promoción social constituye un débil sustituto del empleo que hace que las personas se encuentren "ocupadas", que "hagan algo", mientras se encuentran sin trabajo. Se trata, pues, de un ámbito que presenta un escaso valor social y que, a la vez, ofrece menores posibilidades de formación laboral que aquellos a las que pueden acceder y poseen otros sectores sociales mejor situados socialmente.

Asimismo, resulta conveniente destacar que la formación ofrecida en estos espacios formativos es señalada por algunas de las personas inmigrantes que a ellos asisten como una posibilidad de obtener 
información práctica, valorada como eminentemente positiva, más allá de que no ofrezcan una posibilidad cierta de encontrar trabajo.

\section{Nuevas preguntas para otras partidas}

Desgraciadamente, la fragilidad y vulnerabilidad de la organización del sistema de formación para la inserción laboral de las personas inmigrantes se ha visto reconfirmada de modo inesperado por acontecimientos recientes. Si bien, tal como hemos señalado en la tesis, los procesos de privatización de derechos sociales ya estaban claramente presentes desde hace largo tiempo, el ritmo adquirido a finales de este trabajo se ha incrementado de una manera inicialmente no prevista.

Recientes actuaciones políticas recogidas ampliamente en los medios de comunicación lo confirman. Citemos tan sólo como ejemplos las drásticas reducciones en el presupuesto a la formación laboral, donde se han quitado fondos de formación a sindicatos para ser destinados a "emprendedores que crean empleo", y los anuncios o planes de privatización de los servicios de desempleo en algunas comunidades autónomas.

Este proceso indica un cambio significativo en la forma de consideración de la formación como estrategia de intervención sociopolítica. Por un lado, confirma el escaso valor social que los actuales gobiernos le otorgan más allá de su siempre proclamada importancia, y, por otro, ahonda en el abandono de la acción mediadora estatal en el ámbito de la formación para el empleo. Si la formación era un débil sustituto del empleo, en un momento de índices abismales de desocupación, que oficialmente pasan del $25 \%$ de la población activa, ahora surge la pregunta acerca de cuál será la estrategia o estrategias de sustitución ante la falta de recursos de formación.

Para finalizar este recorrido queremos señalar tres nuevos interrogantes que emergen del proceso de realización de la tesis y que, yendo desde la pregunta más general a la más particular, pueden constituir puntos de partida para nuevas indagaciones.

\section{¿Qué papel se atribuye y debe atribuir al Estado y al bienestar social colectivo?}

El Estado de bienestar ha tenido un preciso desarrollo socio-geo-histórico que ha hecho que en su devenir surgieran configuraciones en las que se garantizó la membresía social a partir de protecciones sociales que reemplazaban otras formas de solidaridad y que ofrecían un particular tipo de pertenencia mediante tales protecciones y derechos.

Una vez que se ha comprobado la persistencia de la capacidad del Estado de producir categorías y de articular tales categorías con derechos que éste concede o niega, y, paralelamente habiendo abandonado activamente el resguardo de los derechos sociales de los ciudadanos y residentes, resulta una tarea pendiente repensar el papel que éste tiene en la creación y/o fomento del bienestar colectivo.

Como ya hemos indicado, en nuestro trabajo hemos recurrido al uso de comillas cuando hablábamos del Estado de bienestar, para indicar la desaparición de las condiciones sociales que harían posible hablar de Estado de bienestar sin más. Sin embargo, la dinámica de los procesos que la tesis pudo reconocer e identificar ha adquirido tal extensión e intensidad que hoy en día, especialmente en el seno de algunos movimientos sociales, es común hablar de Estado "del malestar". 
En este sentido, consideramos que continúa vigente y es más necesario que nunca poner en discusión los modos en los que la intervención del Estado promueve (o, en su omisión, corroe) lazos de pertenencia entre los integrantes de una determinada formación sociopolítica; es decir, discutir desde qué valores, marcos legales y derechos sociales el Estado podría promover nuevas socialidades que rehuyan el menosprecio entre sus integrantes.

En este sentido, se trataría de indagar acerca de la capacidad del Estado para favorecer la creación de las condiciones necesarias para la emergencia de sociabilidades alternativas basadas en derechos donde tengan lugar diferentes actores sociales con sus semejanzas y diferencias. Queda, entonces, la pregunta abierta en sus dimensiones teóricas, sociales y políticas acerca de la capacidad estatal no sólo para reinventarse y ser reinventado, al decir de Boaventura de Sousa Santos (2005), como además para promover el bienestar social colectivo.

\section{¿Qué prácticas de intervención formativamente sociopolíticas llevar a cabo?}

Durante la realización de la tesis se ha podido constatar cómo a través de las prácticas de intervención dirigida a personas inmigrantes se conforman determinados sujetos políticos. En un contexto de remodelación del Estado cabe preguntarse qué tipo de prácticas de intervención se requieren para alcanzar procesos de formación que eviten generar efectos en sentido contrario a los objetivos originalmente planteados. Un esbozo de respuesta iría en la línea de insistir en la importancia de la participación colectiva de las personas que llevan a cabo procesos formativos en cuanto al propio diseño y planificación de los contenidos formativos en colaboración con actores que puedan llevar adelante tales propuestas para vehiculizar concretamente las alternativas prefiguradas.

De cara a concebir los procesos formativos como procesos colectivos, consideramos que los diferentes actores sociales deberían implicarse para lograr alternativas formativas efectivas que superen la perspectiva cortoplacista y que no respondan sólo a los intereses de uno de los actores comprometidos en dicho el proceso. De tal modo se podría dar cabida a unas prácticas de empoderamiento tanto a nivel formativo como a nivel ciudadano.

\section{¿Qué objetos de estudio investigar?}

La tesis tuvo por desafío sistematizar cursos y servicios que se caracterizan por ser inestables y cambiantes. Dichos rasgos dan prueba del escaso valor social que se les concede, un valor que disminuirá aún más en el caso de aquellos recursos que se dirigen a inmigrantes en situación administrativa irregular; y, por ello, considerados usuarios "ilegítimos".

Abdelmalek Sayad (2010) apunta que el valor social de aquello que se estudia se traduce en la valoración social de las investigaciones que asumen estudiarlo. En este sentido, todos aquellos trabajos que pretendiendo conocer los procesos inmigratorios no apunten a "resolver" el problema que la inmigración representaría desde la óptica de la sociedad de instalación, parecería no tener cabida en las agendas de investigación. Desde el punto de vista que hemos asumido resulta, por el contrario, fundamental seguir investigando colectivamente sobre objetos poco estudiados y subteorizados, como es el caso que aquí he pretendido estudiar, realizando cruces temáticos poco explorados, pero a la vez muy iluminadores de los desafíos a los que nos encara el presente. En esta operación es preciso quebrantar las normas dominantes que de modo más o menos explícito definen qué investigaciones son posibles, necesarias y valiosas; deseables, en una palabra. Una mirada transdisciplinar, que además 
esté abierta a lo adisciplinar, se nos ha presentado como indispensable en la elaboración de la tesis, aunque pueda ser considerada como eclécticamente transgresora de esas fronteras disciplinares que garantizarían la seriedad y cientificidad de las investigaciones. No obstante, es preciso otorgar la "prioridad al objeto" para poder conocerlo y reconocerlo, arriesgándonos incluso a perder las garantías ofrecidas por los "hogares" disciplinares.

Concluyamos este apartado diciendo que la alteración de los fundamentos sociopolíticos que se han dado en el desarrollo de una forma de establecer y mantener vínculos sociales marcan la profunda incertidumbre que contiene la interrogación por los criterios de membresía sociopolítica. Esta cuestión evidentemente no es meramente académica y, en todo caso, da cuenta de la insuficiencia que caracteriza a los esfuerzos llevados hasta el momento en la consecución de la igualdad y el reconocimiento sociales. Las cuestiones aquí tratadas siguen abiertas, como así también la necesidad de no concebir los trazos señalados como inevitables, sino que, por el contrario, es preciso poder recuperar los caminos hechos, recorridos, sin rehuir esas nuevas posibilidades cada vez más indispensables y urgentes para la constitución de nuevas, más democráticas y justas socialidades.

En suma, y para finalizar con una pregunta que de manera sintética reformule la pregunta-guía que se ha constituido e intentado responder a lo largo de la tesis, quedando así como una tarea necesariamente inconclusa y a seguir encarando colectivamente en el campo del pensamiento y la investigación social, así como en el del debate y las prácticas políticas: ¿qué nuevos objetos y perspectivas de estudio nos permitirán conocer mejor los nuevos papeles que el Estado adopta, así como las nuevas prácticas de formación sociopolíticas que se ponen en obra, y ello de cara a poder reinventar democráticamente uno y otras?

\section{Referencias}

Aparicio Gómez, Rosa (2002). El Estado de bienestar y la inmigración en España. Madrid: IMSERSO Instituto de Migraciones y Servicios Sociales.

Bourdieu, Pierre (1999a). Contrafuegos. Reflexiones para servir a la resistencia contra la invasión neoliberal. Barcelona: Anagrama.

Bourdieu, Pierre (Dir.) (1999b). La miseria del mundo. Madrid: Akal.

Bourdieu, Pierre (2001). Contrafuegos 2. Por un movimiento social europeo. Barcelona: Anagrama.

Bourdieu, Pierre (2012). L'état. Paris: Raisons d'Agir.

Cachón, Lorenzo (2009). La España inmigrante: marco discriminatorio, mercado de trabajo y políticas de integración. Barcelona: Anthropos.

Cachón, Lorenzo y Laparra, Miguel (Eds.) (2009). Inmigración y políticas sociales. Barcelona: Ed. Bellaterra.

Clavijo, Claudia y Aguirre, Mariano (Eds.) (2002). Políticas Sociales y Estado de bienestar en España. Las migraciones: informe 2002. Madrid: Fundación Hogar del Empleado.

Garde, Juan Antonio (2000). Políticas sociales y estado de bienestar en España: informe 2000. Madrid: Trotta-Fundación Hogar del Empleado.

Geddes, Andrew (2006). Inmigración y Estado de Bienestar en Europa. Zona Abierta, 116-117, 171-190.

Geddes, Andrew y Bommes, Michael (Eds.) (2000). Immigration and Welfare: challenging the borders of the Welfare State. London: Routledge.

Moreno Fuentes, Francisco Javier y Bruquetas Callejo, María (2011). Immigració i Estat del Benestar a Espanya. Barcelona: Obra Social Fundació "La Caixa". 
Santos, Boaventura de Sousa (2005). El Milenio huérfano: ensayos para una nueva cultura política. Madrid: Trotta.

Sayad, Abdelmalek (2010). La doble ausencia: de las ilusiones del emigrado a los padecimientos del inmigrado. Barcelona: Anthropos.

Shore, Cris y Wright, Susan (Eds.) (1997). Anthropology of policy. Critical perspectives on governance and power. London : Routledge.

Este texto está protegido por una licencia Creative Commons.
Usted es libre de copiar, distribuir y comunicar públicamente la obra bajo las siguientes condiciones:
Reconocimiento: Debe reconocer y citar al autor original.
No comercial. No puede utilizar esta obra para fines comerciales.
Sin obras derivadas. No se puede alterar, transformar, o generar una obra derivada a partir de esta obra.
Resumen de licencia - Texto completo de la licencia

